\title{
Effect of Chronic Exposure to Antipsychotic Medication on Cell Numbers in the Parietal Cortex of Macaque Monkeys
}

\author{
Glenn T Konopaske', Karl-Anton Dorph-Petersen ${ }^{1,2}$, Joseph N Pierri', Qiang Wu ${ }^{3}$, Allan R Sampson ${ }^{3}$ \\ and David A Lewis*, I,4 \\ 'Department of Psychiatry, University of Pittsburgh, Pittsburgh, PA, USA; ${ }^{2}$ Center for Basic Psychiatric Research, Aarhus University Psychiatric \\ Hospital, Arhus, Denmark; ${ }^{3}$ Department of Statistics, University of Pittsburgh, Pittsburgh, PA, USA; ${ }^{4}$ Department of Neuroscience, University of \\ Pittsburgh, Pittsburgh, PA, USA
}

\begin{abstract}
Both in vivo and post-mortem investigations have demonstrated smaller volumes of the whole brain and of certain brain regions in individuals with schizophrenia. It is unclear to what degree such smaller volumes are due to the illness or to the effects of antipsychotic medication treatment. Indeed, we recently reported that chronic exposure of macaque monkeys to haloperidol or olanzapine, at doses producing plasma levels in the therapeutic range in schizophrenia subjects, was associated with significantly smaller total brain weight and volume, including an 11.8-15.2\% smaller gray matter volume in the left parietal lobe. Consequently, in this study we sought to determine whether these smaller volumes were associated with lower numbers of the gray matter's constituent cellular elements. The use of point counting and Cavalieri's principle on Niss-stained sections confirmed a 14.6\% smaller gray matter volume in the left parietal lobe from antipsychotic-exposed monkeys. Use of the optical fractionator method to estimate the number of each cell type in the gray matter revealed a significant 14.2\% lower glial cell number with a concomitant 10.2\% higher neuron density. The numbers of neurons and endothelial cells did not differ between groups. Together, the findings of smaller gray matter volume, lower glial cell number, and higher neuron density without a difference in total neuron number in antipsychotic-exposed monkeys parallel the results of post-mortem schizophrenia studies, and raise the possibility that such observations in schizophrenia subjects might be due, at least in part, to antipsychotic medication effects.
\end{abstract}

Neuropsychopharmacology (2007) 32, I216-1223. doi:I0.1038/sj.npp. I 301233; published online 25 October 2006

Keywords: macaque monkeys; haloperidol; olanzapine; schizophrenia; stereology

\section{INTRODUCTION}

Both structural imaging (Shenton et al, 2001) and postmortem (Harrison, 1999) studies have revealed smaller volumes of the whole brain and of certain cortical regions in schizophrenia subjects. Although smaller regional brain volumes have been observed in antipsychotic-naive individuals with first-episode schizophrenia (Gur et al, 1998; Keshavan et al, 1998; Szeszko et al, 2003), some longitudinal imaging studies suggest that brain volume declines further after the initiation of antipsychotic medication treatment. For example, Gur et al (1998) observed that in antipsychotic-naive individuals with first-episode schizophrenia, the volumes of the frontal and temporal lobes decreased

*Correspondence: Dr DA Lewis, Department of Psychiatry, University of Pittsburgh, 38 II O'Hara Street, WI 650 BST Biomedical Science Tower, Pittsburgh, PA 15213, USA, Tel: + I 412624 3934; Fax: + | 412624 99|0, E-mail: lewisda@upmc.edu

Received 8 May 2006; revised 29 August 2006; accepted 15 September 2006

Online publication: 19 September 2006 at http://www.acnp.org/ citations/Npp091906060304/default.pdf significantly after 1.5 years of antipsychotic medication treatment. In addition, smaller whole-brain volumes (Wood et al, 2001) and larger cerebral ventricle volumes (Lieberman et al, 2001) were also observed in first-episode schizophrenia subjects after antipsychotic medication treatment for up to 2 years. Since these imaging studies appropriately did not include healthy control subjects exposed to antipsychotic medications, it is not possible to determine if the changes in brain volumes reflected the underlying disease process and/or the effects of antipsychotic medications. Interpretations of volumetric findings from post-mortem schizophrenia studies are similarly constrained.

Although limited in other respects, the interpretation of schizophrenia studies can be informed through the study of macaque monkeys chronically exposed to antipsychotic medications under controlled conditions. For example, we recently reported that chronic exposure to haloperidol or olanzapine was associated with smaller brain weight and volume in macaque monkeys (Dorph-Petersen et al, 2005). The mean volume of the left cerebrum was significantly smaller by $8.8 \%$ in the haloperidol group and by $10.5 \%$ in 
the olanzapine group relative to the sham group. Among the five brain areas assessed, the left parietal lobe exhibited one of the greatest volume decreases. It was significantly smaller by $11.1 \%$ in the haloperidol group and by $13.3 \%$ in the olanzapine group. In addition, the gray matter volume in the left parietal lobe was also significantly smaller in both the haloperidol (11.8\%) and olanzapine (15.2\%) groups. The parietal white matter volume was also smaller in both the haloperidol (13.3\%) and olanzapine (12.7\%) groups relative to the sham group; however, neither difference achieved statistical significance.

Although these findings require replication, they raise the question of whether a smaller gray matter volume in a certain brain region of antipsychotic-exposed monkeys might be associated with lower numbers of one or more of the gray matter's constituent cellular elements. Because of the magnitude of the volume reduction in the parietal lobe, it offered the best opportunity to answer this question. In addition, the answer to this question might be particularly informative given the findings that, in at least some cortical regions, schizophrenia is associated with lower glial cell number (Hof et al, 2003; Stark et al, 2004) without a change in neuron number (Pakkenberg, 1993; Thune et al, 2001). In order to address this question, an unbiased stereological design was used to estimate the total numbers and densities of neuronal, glial, and endothelial cells in the gray matter of the left parietal lobe from the same cohort of monkeys.

\section{MATERIALS AND METHODS}

\section{Antipsychotic Drug Administration to Monkeys}

The procedures for the chronic exposure of macaque monkeys to antipsychotic medications, euthanasia, brain removal, and dissection were reported previously (DorphPetersen et al, 2005). In brief, 18 experimentally naive, sexually mature (4.5-5.3 years of age) male macaque monkeys (Macaca fascicularis) were divided into three groups ( $n=6$ monkeys/group). In addition, mean body weight was balanced across experimental groups. All studies were carried out in accordance with the NIH Guide for the Care and Use of Laboratory Monkeys and were approved by the University of Pittsburgh Institutional Animal Care and Use Committee.

The monkeys were trained to self-administer, twice daily, custom-made sucrose pellets (Research Diets Inc., New Brunswick, NJ) containing haloperidol sulfate (Spectrum Chemicals and Laboratory Products, Gardena, CA), olanzapine (Eli Lilly, Indianapolis, IN), or no antipsychotic medication (sham). The doses (mean) of haloperidol and olanzapine were titrated to $24-28 \mathrm{mg}(27.3 \mathrm{mg})$ per day for haloperidol and 11-13.2 mg (11.9 mg) per day for olanzapine. These doses produced a mean steady-state trough plasma level of $\sim 1.5 \mathrm{ng} / \mathrm{ml}$ for haloperidol and $\sim 15 \mathrm{ng} / \mathrm{ml}$ for olanzapine. These plasma levels have been associated with antipsychotic efficacy in individuals with schizophrenia, while minimizing extrapyramidal symptoms and hyperprolactinemia (Kapur et al, 1997, 1998; Oosthuizen et al, 2004; Perry et al, 2001). The monkeys in the haloperidol group received haloperidol for a mean of 27 months. However, due to procurement delays, monkeys in the olanzapine group received sham pellets for approximately 10 months before receiving olanzapine for a mean of 17 months. Monkeys had free access to water, and ate 20-30 food pellets (Purina Monkey Chow) daily following the afternoon drug administration. The mean weight gains in the haloperidol and olanzapine groups were not different from the sham. In addition, both initial and terminal mean body weights were not different across groups (Dorph-Petersen et al, 2005).

\section{Tissue Processing}

Following antipsychotic medication exposure, monkeys were matched by terminal body weight and euthanized in triads. The brain was extracted, and the cerebellum and brainstem were removed by cutting through the mesencephalon at the level of the superior colliculi. The cerebral hemispheres, cerebellum, and brainstem were bisected, and the left cerebrum was dissected, following gyration patterns, producing five pieces: (1) frontal; (2) parietal; (3) occipital; (4) temporal; and (5) cerebellum and brainstem. The parietal piece was defined rostrally by the bottom of the central sulcus, caudally by a planar cut through the lunate sulcus extending to the medial surface, and ventrally by a cut from the bottom of the lateral fissure extending through the dorsal edge of the corpus callosum and by a cut from the bottom of the lateral fissure through the angular gyrus. All pieces were placed in $4 \%$ paraformaldehyde for $48 \mathrm{~h}$, rinsed in a graded series of sucrose solutions, and stored in cryoprotectant at $-30^{\circ} \mathrm{C}$. For each monkey, the interval from the extraction of the brain to the immersion of the pieces in paraformaldehyde was below $72 \mathrm{~min}$.

Each parietal piece was embedded in 7\% low-melt agarose (SeaPlaque Agarose, Cambrex, Rockland, ME) and cut (perpendicular to the intraparietal sulcus) in a systematic and uniformly random manner producing 12-15 slabs with a mean thickness of $T=2.5 \mathrm{~mm}$. Each monkey was assigned a coded number and the position of monkeys from each experimental group was randomized within individual triads. Each slab was mounted using the Precision Cryoembedding system (Pathology Innovations, Wyckoff, NJ) (Peters, 2003a, b) and four $80 \mu \mathrm{m}$ sections (ie, the block advance, $B A=80 \mu \mathrm{m}$ ), containing the full face of the slab, were cut from the rostral surface on a cryostat. With a random start, the same numbered section was selected from each slab in a triad and the section number was shifted by one in each subsequent triad. The resulting sets of systematic and uniformly random sections were immersed in $4 \%$ paraformaldehyde for $24 \mathrm{~h}$, mounted, dried overnight, and stained for Nissl substance with thionin.

\section{Stereological Assessment of Parietal Volumes}

A single investigator (GTK), blinded to experimental group and subject number, conducted all observations on the Nissl-stained sections using an Olympus BX51 microscope equipped with a MT1201 microcator $(0.2 \mu \mathrm{m}$ resolution), an ND281B readout (Heidenhain, Germany), and a X-Y-Z motorized specimen stage (ProScan, Prior Scientific, UK). Using a $\times 2$ photo eyepiece (PE2X, Olympus, Japan), a three-chip CCD camcorder (KY-F55B, JVC, Japan) was mounted on the top of the microscope, and forwarded a $760 \times 570$ pixel live image (50 frames/s) to a personal 
computer. The computer ran the CAST stereology software package (Version 2.00.07, Olympus, Denmark), and was fitted with a frame grabber (Flashpoint 3D, Integral Technologies, IN) and a 19 inch monitor (FlexScan T765 Color Display Monitor, EIZO Nanao Corp., Japan) having a screen resolution of $1280 \times 1024$ pixels. The microscope was calibrated daily using a calibration slide, and was mounted on a vibration isolation table (Q500A, Qontrol Devices Inc., CA) to facilitate high-magnification imaging.

Point counting was carried out on each Nissl-stained section using a Plan Apo $\times 1.25$ objective and a final magnification of $\times 33$ at the monitor. A grid of points (area per point, $a=2.36 \mathrm{~mm}^{2}$ ) was superimposed over each section and an average of 403 points were counted in the gray matter. From these counts, and using the Cavalieri method (Gundersen and Jensen, 1987; Howard and Reed, 1998), the gray matter volume of each parietal piece was estimated as: $V:=\operatorname{Ta} \Sigma P_{i}$, where $T$ is the mean slab thickness, $a$ is the area per point, and $P_{i}$ is the number of points hitting the gray matter. Here and elsewhere ':=' indicates 'estimated by'.

\section{Stereological Assessment of Total Cell Numbers in the Parietal Cortex}

A Plan Apo $\times 4$ objective was used to draw contours around the gray matter in each Nissl-stained section. Using the nucleus as the sampling item, all nucleated cells were counted in optical disectors (Gundersen, 1986) using a Plan Apo $\times 100$ oil-immersion objective $(\mathrm{NA}=1.4)$ at a final magnification of $\times 2677$ at the monitor. In addition, counted cells were subtyped based on morphological criteria (Figure 1). Neurons had euchromatin in the nucleus, a clearly visible nucleolus, and surrounding cytoplasm. Glial cells had heterochromatin in the nucleus and no cytoplasm. Endothelial cells had a curved shape or were located around a blood vessel. Cells not otherwise classified were marked as 'unknown'. No efforts were made to distinguish among

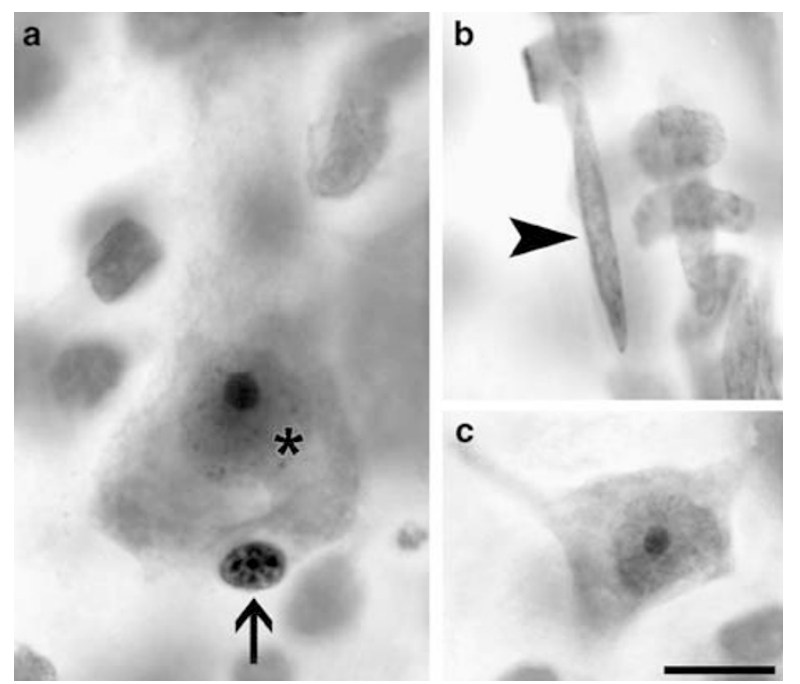

Figure I Bright field photomicrographs demonstrating the distinguishing morphology of neuronal, glial, and endothelial cells. (a) Neuronal (pyramidal) cell (*), and glial cell (arrow). (b) Endothelial cell (arrowhead). (c) neuronal (multipolar interneuron) cell. Calibration bar $=25 \mu \mathrm{m}$. classes of neuronal or glial cells. To assess for intrarater reliability of cell classification, 12 sections were arbitrarily selected from different monkeys. Cells were counted through the full thickness of each section twice on different days. The intraclass correlation coefficients for intrarater reliability across sections were 0.94 for neurons, 0.94 for glia, and 0.79 for endothelial cells.

In an initial calibration study, cells were sampled using unbiased counting frames (Gundersen, 1977) in the full thickness of 30 sections that were chosen from all monkeys in a systematic and uniformly random manner. A total of 2178 cells were counted, the $z$-position (ie, the distance from the section surface) of each counted cell was recorded, and section thickness was measured at every frame containing a counted cell. These calibration data demonstrated that shrinkage in section thickness was linear (Dorph-Petersen et al, 2001, 2004b) and that lost caps at the surfaces of the sections had a limited impact.

Based on the data from the calibration study, the optical fractionator (West et al, 1991) was used to estimate the total numbers of neuronal, glial, and endothelial cells in the gray matter of the left parietal lobe from all monkeys using an unbiased counting frame with an area of $a_{\text {frame }}=219.7 \mu \mathrm{m}^{2}$, a disector height of $h=8 \mu \mathrm{m}$, and an upper guard zone of $2.9 \mu \mathrm{m}$. The distance between frames (ie, the 'stepping distance'), $D$, was kept constant for all the sections within each monkey, but varied from 700 to $800 \mu \mathrm{m}$ across monkeys. $D$ was adjusted for each monkey using the point count-based volume estimates and mean cell densities observed in the previous monkeys (calibration values were used for the first monkey) to achieve counts above 600 for both neuronal and glial cells. As a result, an average of 948 neuronal, 767 glial, 299 endothelial, and 86 unknown cells (2099 all cell types) were counted in each monkey.

Similar to the calibration study, section thickness was measured at each frame containing a counted cell. Mean section thickness across all sections was $20.5 \mu \mathrm{m}$ with a mean coefficient of variation $(C V)$ of 0.15 within each monkey and a mean $C V$ of 0.06 across all monkeys. Thus, despite a marked shrinkage of $\sim 75 \%$ in section thickness and a high degree of variability in thickness within sections, mean section thickness was stable across monkeys.

Uneven shrinkage in section thickness can introduce biases when using the classical optical fractionator. However, such potential biases were eliminated by using the optical fractionator based on a mean section thickness that was number weighted $\left(\bar{t}_{Q^{-}}\right)$(Dorph-Petersen et al, 2001, 2004b). Mean section thickness was number weighted as follows:

$$
\bar{t}_{Q^{-}}=\sum_{i}\left(t_{i} q_{i}^{-}\right) / \sum_{i} q_{i}^{-}
$$

where $t_{i}$ is the local section thickness in the center of the $i$ th counting frame having a count of $q_{i}^{-}$(Dorph-Petersen et al, 2001). Total cell numbers were estimated as:

$$
N:=\frac{1}{a s f} \frac{1}{s s f} \frac{1}{h s f} \sum Q^{-}
$$

where asf is the area sampling fraction $\left(a_{\text {frame }} / D^{2}\right), s s f$ is the section sampling fraction $(B A / T), h s f$ is the height sampling fraction $\left(h / \bar{t}_{Q^{-}}\right)$, and $Q^{-}$is the number of a given cell type counted. 


\section{Precision of the Stereological Estimates}

Previously published measurements of the left parietal lobe volume in these monkeys were made using an implementation of Archimedes' principle of water displacement (Scherle, 1970). These measurements were very precise and had a mean coefficient of error $(C E)$ of $\sim 0.004$ (DorphPetersen et al, 2005). Unbiased stereological estimates made from tissue sections, such as those in the present study, are typically associated with a higher level of error and thus are less precise. In many stereological studies, this problem is addressed by keeping the variance due to measurement error somewhat lower than the variance due to intrinsic biology. Consequently, the most efficient way to increase a stereological study's statistical power is by increasing subject number (Gundersen and Østerby, 1981). However, in this study the number of available monkeys was limited. In addition, power calculations suggested that a high level of precision in the stereological estimates might reveal potential differences between experimental groups. Thus, we used a large number of sections ( $\sim 13$ per monkey) and cell counts for neurons and glial cells $(\sim 800$ of each per monkey) to produce stereological estimates with a high precision (Table 1), albeit at the cost of increasing microscopy time. The precision of the volume estimates were calculated using the methods of Gundersen and Jensen (1987) and Gundersen et al (1999), and the precision of the number estimates were calculated using the methods of Gundersen et al (1999). Owing to the stereological estimates' high precision, $\sim 90 \%$ of the total observed variance within groups is due to variability in intrinsic biology across monkeys. Additional variance was introduced into the estimates because some degree of uncertainty is inherent in classifying cells; however, this additional variance is likely quite small as over $95 \%$ of cells were readily classified.

In addition to the (very low) stereological measurement $C E$ assessed above, the precision of the final estimates are influenced by the precision of the parietal lobe's delineation. Our approach did not produce a systematic bias since the positions of the cuts that defined the parietal lobe were dictated by the cortical gyration pattern within each individual brain, and we do not expect the gyration pattern or the position of cytoarchitectonic boundaries to be influenced by antipsychotic exposure. In addition, the direction of the volume changes was similar in all five dissected brain regions from the antipsychotic-exposed monkeys relative to the sham monkeys (Dorph-Petersen et al, 2005), indicating that the smaller parietal volumes in the antipsychotic-exposed animals cannot be explained by a differential dissection across animal groups. Our approach also has the added strength of ensuring that a brain region's delineation remains constant across measurements (eg, volume estimates $v s$ cell number estimates). Furthermore, the use of cytoarchitectonic criteria does not increase the precision of brain region delineation because, as we have shown in previous studies, the determination of cytoarchitectonic boundaries is associated with variability that limits precision (Dorph-Petersen et al, 2004a; Sweet et al, 2005).

\section{Statistical Analyses}

A two-way ANOVA model, with group as the main effect and triad as a blocking factor, was used to assess the effect of chronic antipsychotic exposure on the gray matter volume in the left parietal lobe from macaque monkeys. Post hoc comparisons of volume between groups were made using Tukey's multiple comparisons test. Because volume data, obtained previously (Dorph-Petersen et al, 2005) and in the current study, indicated that the haloperidol and olanzapine groups both differed from the sham group in the same direction and to a similar magnitude, the contrast of the combined antipsychotic-exposed group $v s$ the sham group, based upon a two-way ANOVA model, was used to assess the effect of chronic antipsychotic exposure on the cell number and density data. One-tailed testing of these contrasts was carried out due to the directionality of the hypothesized effect (eg, reduced cell number in antipsychotic-exposed monkeys). Analyses were implemented in SPSS and SAS PROC GLM with $\alpha=0.05$.

\section{RESULTS}

Consistent with previous measures that were highly precise (Dorph-Petersen et al, 2005), mean gray matter volume in the left parietal lobe differed significantly across

Table I Summary of Stereological Results

\begin{tabular}{|c|c|c|c|c|c|}
\hline & $N_{\text {all }}\left(\times 10^{6}\right)(C E)$ & $N_{\text {neuron }}\left(\times 10^{6}\right)(C E)$ & $N_{\text {glia }}\left(\times 10^{6}\right)(C E)$ & $N_{\text {endothelial }}\left(\times 10^{6}\right)(C E)$ & $N_{\text {unknown }}\left(\times 10^{6}\right)(C E)$ \\
\hline \multicolumn{6}{|l|}{ Sham } \\
\hline Mean & $460(0.02)$ & $202.1(0.03)$ & I $75.3(0.04)$ & $64.7(0.06)$ & $18(0.12)$ \\
\hline $\mathrm{SD}$ & 58.6 & 24.6 & 33.3 & 18 & 6.7 \\
\hline$C V$ & 0.13 & 0.12 & 0.19 & 0.28 & 0.37 \\
\hline Mean & $418.6(0.02)$ & $190.1(0.03)$ & I $50.3(0.04)$ & $60.7(0.06)$ & $17.4(0.11)$ \\
\hline $\mathrm{SD}$ & 35.6 & 24.8 & 11.4 & II & 4 \\
\hline $\mathrm{CV}$ & 0.09 & 0.13 & 0.08 & 0.18 & 0.23 \\
\hline
\end{tabular}

$N_{\text {all }}$ is the total number of all cells, $N_{\text {neuron }}$ is neuron number, $N_{\text {glia }}$ is glial number, $N_{\text {endothelial }}$ is endothelial number, and $N_{\text {unknown }}$ is unknown number. Standard deviation (SD), mean coefficient of error (CE), and interindividual coefficient of variation (CV) are listed for all measures. 
experimental groups $\left(\mathrm{F}_{(2,10)}=7.11, p=0.012\right)$. Gray matter volume was significantly smaller by $13.9 \%(p=0.03)$ in the haloperidol group and by $15.3 \%(p=0.02)$ in the olanzapine

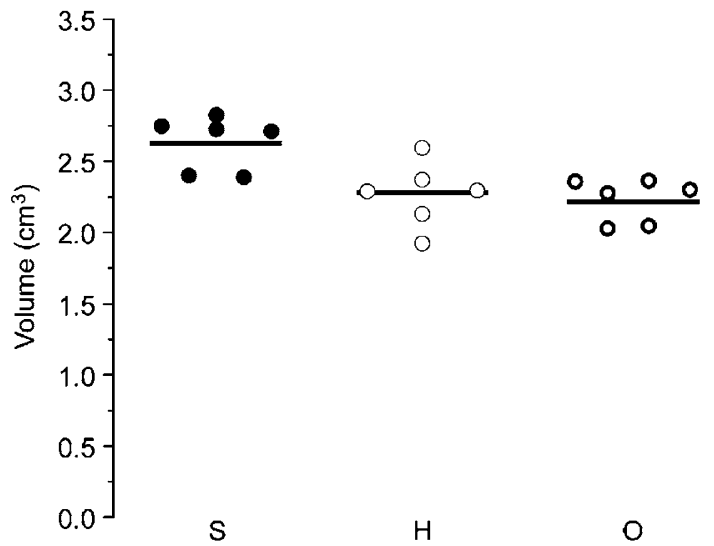

Figure 2 Left parietal gray matter volumes for the sham- (S), haloperidol- $(\mathrm{H})$, and olanzapine- $(\mathrm{O})$ exposed monkeys. Significant differences were found between haloperidol and sham $(p=0.03)$ and olanzapine and sham $(p=0.02)$, but not between haloperidol and olanzapine $(p=0.95)$ groups. The horizontal bars indicate group means.

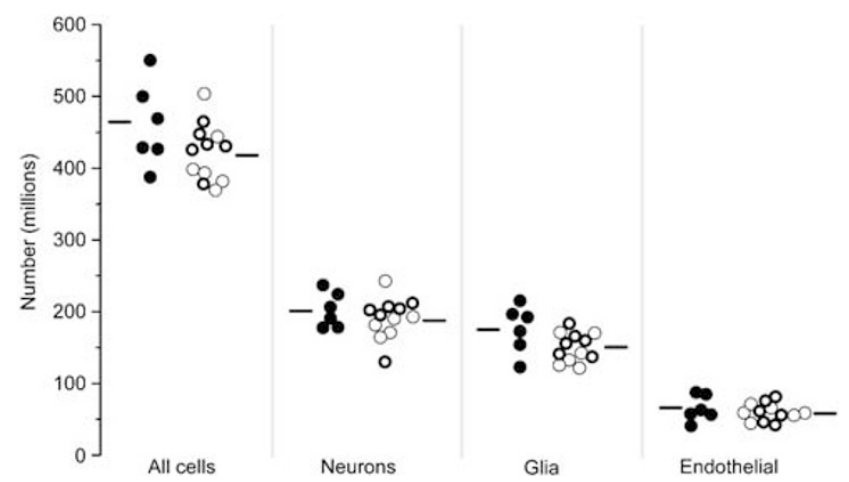

Figure 3 Cortical cell numbers from the left parietal lobe of the haloperidol- (thin open circles), olanzapine- (thick open circles), and sham(filled circles) exposed monkeys. The number of glial cells significantly differed between the antipsychotic-exposed and sham groups $(p=0.04)$, and the total number of all cell types trended toward significance $(p=0.07)$. The horizontal bars indicate sham and antipsychotic-exposed group means. group (Figure 2) relative to the sham group, but did not differ between the haloperidol and olanzapine groups $(p=0.95)$. The mean $C E$ was 0.02 for the estimates of gray matter volume.

In the gray matter of the left parietal lobe from antipsychotic-exposed monkeys, a 9\% lower total number of all cell types trended toward significance $(t=-1.63$, $p=0.07)$. This effect appeared to be largely explained by a significant $(t=-1.93, p=0.04) \quad 14.2 \%$ lower glial cell number (Table 2 and Figure 3 ). In contrast, no reductions were found in the numbers of neuronal $(t=-0.96$, $p=0.18)$, endothelial $(t=-0.72, p=0.24)$, or unknown $(t=-0.26, p=0.4)$ cells. Similar effects were also observed when individual antipsychotic groups were compared against sham (Table 2).

The densities (Figure 4) of all cell types, neurons, and endothelial cells were higher by $7,10.2$ and $10.3 \%$, respectively, in the antipsychotic-exposed monkeys. However, only the densities of all cell types $(t=1.76, p=0.055)$ and neurons $(t=1.75, p=0.055)$ trended toward significance. The densities of glial $(t=0.18, p=0.43)$, endothelial

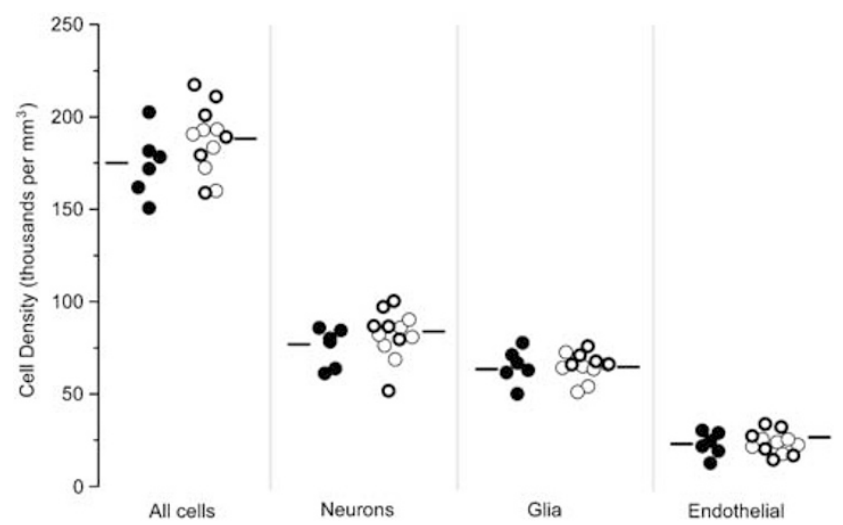

Figure 4 Cortical cell densities from the left parietal lobe of the haloperidol- (thin open circles), olanzapine- (thick open circles), and sham(filled circles) exposed monkeys. The densities of all cell types and neurons were greater in the antipsychotic-exposed groups, and trended toward significance $(p=0.055$ and $p=0.055$, respectively). The horizontal bars indicate sham- and antipsychotic-exposed group means.

Table 2 Comparison of Antipsychotic Groups with Sham

\begin{tabular}{|c|c|c|c|c|c|c|}
\hline & \multicolumn{2}{|c|}{ All antipsychotics } & \multicolumn{2}{|c|}{ Haloperidol } & \multicolumn{2}{|c|}{ Olanzapine } \\
\hline & Mean \% difference & $p$ & Mean \% difference & $p$ & Mean \% difference & $p$ \\
\hline Neuron number & -5.9 & 0.181 & -6.3 & 0.198 & -5.5 & 0.230 \\
\hline Glial cell number & -14.2 & 0.041 & $-|8|$. & 0.030 & -10.3 & 0.127 \\
\hline Total cell density & 7 & 0.055 & 3.9 & 0.206 & 10 & 0.027 \\
\hline Neuron density & 10.2 & 0.055 & 8.3 & 0.124 & 12.2 & 0.051 \\
\hline Glial cell density & । & 0.430 & -4.3 & 0.268 & 6.4 & 0.181 \\
\hline Endothelial cell density & 10.3 & 0.123 & 7.6 & 0.223 & 12.9 & 0.104 \\
\hline
\end{tabular}

p-Values represent the results of a two-way ANOVA model followed by a one-tailed contrast of the indicated antipsychotic group and sham group. 
$(t=1.23, p=0.12)$, and unknown $(t=1.34, p=0.11)$ cells were not significantly different. Similar effects were also observed when individual antipsychotic groups were compared against sham (Table 2). As seen in Table 1, the $C E$ s indicate that the stereological estimates are highly precise. Cells labeled as 'unknown' represented less than 5\% of all cells counted.

\section{DISCUSSION}

Using a different approach in tissue sections, this study, as expected, replicates in the same material, the previous finding of smaller gray matter volume in the left parietal lobe from monkeys chronically exposed to antipsychotic medications (Dorph-Petersen et al, 2005). In addition, the cell counts suggest that lower glial cell number is associated with a smaller gray matter volume in the left parietal lobe. Furthermore, the higher neuron density is consistent with a preserved neuron number in the face of a smaller gray matter volume.

In a prior study, Selemon et al (1999) examined the effects of chronic antipsychotic exposure on cell densities in the dorsolateral prefrontal cortex (area 46) of macaque monkeys. In antipsychotic-exposed monkeys, neuron and glial cell densities were altered in certain lamina, but no significant differences in the entire cortex were observed. Although cortical volume was not assessed, layer $\mathrm{V}$ was wider in antipsychotic-exposed monkeys, but again, no overall differences in cortical thickness were observed. Importantly, the oral doses used in the Selemon et al (1999) study, while comparable to those administered in humans, do not produce trough plasma levels associated with therapeutic efficacy in schizophrenia because of a much faster metabolic rate of antipsychotic medications in monkeys (Dorph-Petersen et al, 2005; Kassahun et al, 1997; Mattiuz et al, 1997). In addition, monkeys were exposed to antipsychotic medications for only 6 months. Thus, it is difficult to determine whether the absence of differences in overall neuron and glial cell densities and cortical thickness in the Selemon study reflects a lower degree of antipsychotic exposure relative to the present study, or differences in the cortical regions examined and the counting methods employed.

Several questions are raised by our findings of smaller cortical volume, lower glial cell number, higher neuron density, and no change in neuron number in the gray matter of the left parietal lobe from antipsychotic-exposed monkeys. First, how might antipsychotic exposure lead to lower glial cell number? Haloperidol might reduce glial cell number, at least in part, by inducing glial cell death. Indeed, haloperidol reduces glioma cell viability (Behl et al, 1995) and induces neuron apoptosis (Ukai et al, 2004) and necrosis (Behl et al, 1995). Conversely, olanzapine promotes the activation of antiapoptotic pathways in rodent frontal cortex (Bai et al, 2004; Fumagalli et al, 2006; Wang et al, 2001) and in pheochromocytoma cells ( $\mathrm{Lu}$ et al, 2004). In addition, olanzapine also protects pheochromocytoma cells from cytotoxic oxidative damage (Wei et al, 2003). Thus, antipsychotic-induced cell death is not a parsimonious explanation for lower glial cell number and higher neuron density in both the haloperidol and olanzapine groups.
Alternatively, exposure to haloperidol or olanzapine might reduce glial cell production. Glial turnover continues in the adult brain (Kaplan and Hinds, 1980; Lawson et al, 1992; McCarthy and Leblond, 1988), during which certain glial cells are eliminated under physiologic conditions through apoptosis (Barres et al, 1992; Krueger et al, 1995). Eliminated cells are replaced by oligodendrocytes or astrocytes produced from progenitor cells in the CNS (Cameron and Rakic, 1991; Kaplan and Hinds, 1980) or by microglia that are produced in the hematopoietic bone marrow and subsequently migrate to the CNS (Eglitis and Mezey, 1997; Rezaie and Male, 1999). Therefore, in the present study, antipsychotic exposure could have impaired the production of oligodendrocytes, astrocytes, or microglia in the face of ongoing elimination, thereby reducing glial cell number. Although two studies suggest that both haloperidol (Kippin et al, 2005) and olanzapine (Kodama et al, 2004) might increase glial cell production in the rodent brain, both used cell density measurements that can be affected by antipsychotic-induced alterations in brain volume. Thus, explaining the mechanism that accounts for our findings requires both replication of this study and additional studies using glia-specific markers to determine if antipsychotic medications affect certain classes of glial cells and whether such effects differ between rodents and primates.

Second, is the lower glial cell number sufficient to account for the associated smaller gray matter volume in the left parietal lobe? Unfortunately, the proportion of the gray matter volume attributable to glial cell processes and myelin sheaths has not been estimated using unbiased stereological methods. Thus, the degree to which lower glial cell number contributes to a smaller gray matter volume remains an open question. In addition to lower glial cell number, reductions in neuropil elements (eg, axonal fibers and terminals, and dendritic arbors and spines), might also contribute to a smaller gray matter volume. Although certain axon terminals appear unaffected in antipsychoticexposed monkeys (Akil et al, 1999; Lewis et al, 2001; Pierri et al, 1999), antipsychotic exposure could reduce the extent of dendritic arbors and the density of dendritic spines (Benes et al, 1985). However, these structures could not be assessed in the current study.

Third, could the current results be explained by the misclassification of cells? The intrarater correlation coefficient for cell classification was 0.94 for neurons, 0.94 for glia, and 0.79 for endothelial cells, suggesting a high degree of consistency in cell classification. Furthermore, since all counts were carried out blind to subject group, any misclassification would be comparable across groups. Several types of cell misclassification could have occurred: (1) misclassification of small neurons as glial cells, (2) misclassification of glial cells as small neurons, or (3) some small neurons misclassified as glial cells and some glial cells misclassified as small neurons. However, if one assumes that only the parietal gray matter volume is different between subject groups and the total number of neurons and glial cells is not different, then all three types of cell misclassification would produce the same results: no change in total neuron and glial cell numbers, and higher neuron and glial densities in the antipsychotic-exposed monkeys relative to the sham monkeys. Since we found no change in 
neuron number, lower glial cell number, higher neuron density, and no change in glial cell density in the antipsychotic-exposed monkeys relative to the sham monkeys, cell misclassification alone cannot account for the current results.

Fourth, to what extent do the effects of antipsychotic exposure in monkeys parallel the results from post-mortem schizophrenia studies? Interestingly, similar to the findings of the present study, post-mortem studies in schizophrenia subjects have revealed smaller volumes of frontal, parietal, and temporal cortices (Pakkenberg, 1993; Selemon et al, 2002), lower glial cell (area 24) (Stark et al, 2004) and oligodendrocyte number (area 9) (Hof et al, 2003), higher neuron density in the dorsolateral prefrontal (area 9), anterior cingulate (area 24), and primary visual (area 17) cortices (Chana et al, 2003; Selemon et al, 1995), and no change in total neuron number in the prefrontal (Thune et al, 2001) or total neocortex (Pakkenberg, 1993) relative to normal comparison subjects. However, whereas treatment with typical antipsychotics has been reported to lead to smaller volumes in certain cortical areas in subjects with schizophrenia (Dazzan et al, 2005; Lieberman et al, 2005), atypical antipsychotics might preserve cortical volume (Dazzan et al, 2005) or slow the rate of volume decreases (Lieberman et al, 2005). Thus, different antipsychotic medications might interact with the underlying disease process producing different patterns of change in cortical volume. Nevertheless, the findings of the current study at least raise the possibility that some previous reports of morphological and cellular alterations in the cerebral cortex of schizophrenia subjects might be confounded by the effects of chronic antipsychotic treatment.

\section{ACKNOWLEDGEMENTS}

We thank Stephen Eggan, Yoshito Mizoguchi, Kelly Wright, Dianne Cruz, Susan Konopaske, and Robert Sweet for helpful comments to this paper and Mary Brady for help with graphics. David Lewis has received research funding from Eli Lilly, Merck, and Pfizer and has served as a consultant to AstraZeneca, Eli Lilly, Merck, Pfizer, and Sepracor. Allan Sampson is a statistical consultant for Johnson \& Johnson Pharmaceutical Research \& Development LLC. The study received support from Eli Lilly and Company and NIH Grants MH45156, MH01945, and MH16804.

\section{REFERENCES}

Akil M, Pierri JN, Whitehead RE, Edgar CL, Mohila C, Sampson AR et al (1999). Lamina-specific alterations in the dopamine innervation of the prefrontal cortex in schizophrenic subjects. Am J Psychiatry 156: 1580-1589.

Bai O, Zhang H, Li XM (2004). Antipsychotic drugs clozapine and olanzapine upregulate bcl-2 mRNA and protein in rat frontal cortex and hippocampus. Brain Res 1010: 81-86.

Barres BA, Hart IK, Coles HS, Burne JF, Voyvodic JT, Richardson WD et al (1992). Cell death and control of cell survival in the oligodendrocyte lineage. Cell 70: 31-46.

Behl C, Rupprecht R, Skutella T, Holsboer F (1995). Haloperidolinduced cell death - mechanism and protection with vitamin $\mathrm{E}$ in vitro. Neuroreport 7: 360-364.
Benes FM, Paskevich PA, Davidson J, Domesick VB (1985). Synaptic rearrangements in medial prefrontal cortex of haloperidol-treated rats. Brain Res 348: 15-20.

Cameron RS, Rakic P (1991). Glial cell lineage in the cerebral cortex: a review and synthesis. Glia 4: 124-137.

Chana G, Landau S, Beasley C, Everall IP, Cotter D (2003). Twodimensional assessment of cytoarchitecture in the anterior cingulate cortex in major depressive disorder, bipolar disorder, and schizophrenia: evidence for decreased neuronal somal size and increased neuronal density. Biol Psychiatry 53: 1086-1098.

Dazzan P, Morgan KD, Orr K, Hutchinson G, Chritnis X, Suckling J et al (2005). Different effects of typical and atypical antipsychotics on grey matter in first episode psychosis: the AESOP study. Neuropsychopharmacology 30: 765-774.

Dorph-Petersen KA, Nyengaard JR, Gundersen HJ (2001). Tissue shrinkage and unbiased stereological estimation of particle number and size. J Microsc 204: 232-246.

Dorph-Petersen KA, Pierri JN, Perel JM, Sun Z, Sampson AR, Lewis DA (2005). The influence of chronic exposure to antipsychotic medications on brain size before and after tissue fixation: a comparison of haloperidol and olanzapine in macaque monkeys. Neuropsychopharmacology 30: 1649-1661.

Dorph-Petersen KA, Pierri JN, Sun Z, Sampson AR, Lewis DA (2004a). Stereological analysis of the mediodorsal thalamic nucleus in schizophrenia: volume, neuron number and cell types. J Comp Neurol 472: 449-462.

Dorph-Petersen KA, Rosenberg R, Nyengaard JR (2004b). Estimation of number and volume of immunohistochemically stained neurons in complex brain regions. In: Evans SM, Janson AM, Nyengaard JR (eds). Quantitative Methods in Neuroscience. Oxford University Press: Oxford. pp 216-238.

Eglitis MA, Mezey E (1997). Hematopoietic cells differentiate into both microglia and macroglia in the brains of adult mice. Proc Natl Acad Sci USA 94: 4080-4085.

Fumagalli F, Frasca A, Sparta M, Drago F, Racagni G, Riva MA (2006). Long-term exposure to the atypical antipsychotic olanzapine differently up-regulates extracellular signal-regulated kinases 1 and 2 phosphorylation in subcellular compartments of rat prefrontal cortex. Mol Pharmacol 69: 1366-1372.

Gundersen HJ (1977). Notes on the estimation of the numerical density of arbitrary particles: the edge effect. J Microsc 111: 219-223.

Gundersen HJ (1986). Stereology of arbitrary particles. A review of unbiased number and size estimators and the presentation of some new ones, in memory of William R. Thompson. J Microsc 143(Part 1): 3-45.

Gundersen HJ, Jensen EB (1987). The efficiency of systematic sampling in stereology and its prediction. J Microsc 147(Part 3): 229-263.

Gundersen HJ, Jensen EB, Kieu K, Nielsen J (1999). The efficiency of systematic sampling in stereology-reconsidered. J Microsc 193: 199-211.

Gundersen HJ, Østerby R (1981). Optimizing sampling efficiency of stereological studies in biology: or 'do more less well!' J Microsc 121: 65-73.

Gur RE, Cowell P, Turetsky BI, Gallacher F, Cannon T, Bilker W et al (1998). A follow-up magnetic resonance imaging study of schizophrenia. Relationship of neuroanatomical changes to clinical and neurobehavioral measures. Arch Gen Psychiatry 55: $145-152$.

Harrison PJ (1999). The neuropathology of schizophrenia. A critical review of the data and their interpretation. Brain 122(Part 4): 593-624.

Hof PR, Haroutunian V, Friedrich Jr VL, Byne W, Buitron C, Perl DP et al (2003). Loss and altered spatial distribution of oligodendrocytes in the superior frontal gyrus in schizophrenia. Biol Psychiatry 53: 1075-1085. 
Howard CV, Reed MG (1998). Unbiased Stereology: ThreeDimensional Measurement in Microscopy. Bios Scientific Publishers: Oxford.

Kaplan MS, Hinds JW (1980). Gliogenesis of astrocytes and oligodendrocytes in the neocortical grey and white matter of the adult rat: electron microscopic analysis of light radioautographs. J Comp Neurol 193: 711-727.

Kapur S, Zipursky R, Roy P, Jones C, Remington G, Reed K et al (1997). The relationship between D2 receptor occupancy and plasma levels on low dose oral haloperidol: a PET study. Psychopharmacology (Berlin) 131: 148-152.

Kapur S, Zipursky RB, Remington G, Jones C, DaSilva J, Wilson AA et al (1998). 5-HT2 and D2 receptor occupancy of olanzapine in schizophrenia: a PET investigation. Am J Psychiatry 155: 921-928.

Kassahun K, Mattiuz E, Nyhart Jr E, Obermeyer B, Gillespie T, Murphy A et al (1997). Disposition and biotransformation of the antipsychotic agent olanzapine in humans. Drug Metab Dispos 25: 81-93.

Keshavan MS, Rosenberg D, Sweeney JA, Pettegrew JW (1998). Decreased caudate volume in neuroleptic-naive psychotic patients. Am J Psychiatry 155: 774-778.

Kippin TE, Kapur S, van der Kooy D (2005). Dopamine specifically inhibits forebrain neural stem cell proliferation, suggesting a novel effect of antipsychotic drugs. J Neurosci 25: 5815-5823.

Kodama M, Fujioka T, Duman RS (2004). Chronic olanzapine or fluoxetine administration increases cell proliferation in hippocampus and prefrontal cortex of adult rat. Biol Psychiatry 56: 570-580.

Krueger BK, Burne JF, Raff MC (1995). Evidence for large-scale astrocyte death in the developing cerebellum. J Neurosci 15: 3366-3374.

Lawson LJ, Perry VH, Gordon S (1992). Turnover of resident microglia in the normal adult mouse brain. Neuroscience 48: 405-415.

Lewis DA, Cruz DA, Melchitzky DS, Pierri JN (2001). Laminaspecific deficits in parvalbumin-immunoreactive varicosities in the prefrontal cortex of subjects with schizophrenia: evidence for fewer projections from the thalamus. Am J Psychiatry 158: 1411-1422.

Lieberman J, Chakos M, Wu H, Alvir J, Hoffman E, Robinson D et al (2001). Longitudinal study of brain morphology in first episode schizophrenia. Biol Psychiatry 49: 487-499.

Lieberman JA, Tollefson GD, Charles C, Zipursky R, Sharma T, Kahn RS et al (2005). Antipsychotic drug effects on brain morphology in first-episode psychosis. Arch Gen Psychiatry 62: 361-370.

Lu XH, Bradley RJ, Dwyer DS (2004). Olanzapine produces trophic effects in vitro and stimulates phosphorylation of $\mathrm{Akt} / \mathrm{PKB}$, ERK1/2, and the mitogen-activated protein kinase p38. Brain Res 1011: $58-68$.

Mattiuz E, Franklin R, Gillespie T, Murphy A, Bernstein J, Chiu A et al (1997). Disposition and metabolism of olanzapine in mice, dogs, and rhesus monkeys. Drug Metab Dispos 25: 573-583.

McCarthy GF, Leblond CP (1988). Radioautographic evidence for slow astrocyte turnover and modest oligodendrocyte production in the corpus callosum of adult mice infused with ${ }^{3} \mathrm{H}$-thymidine. J Comp Neurol 271: 589-603.

Oosthuizen P, Emsley R, Jadri Turner H, Keyter N (2004). A randomized, controlled comparison of the efficacy and tolerability of low and high doses of haloperidol in the treatment of first-episode psychosis. Int $J$ Neuropsychopharmacol 7: $125-131$.
Pakkenberg B (1993). Total nerve cell number in neocortex in chronic schizophrenics and controls estimated using optical disectors. Biol Psychiatry 34: 768-772.

Perry PJ, Lund BC, Sanger T, Beasley C (2001). Olanzapine plasma concentrations and clinical response: acute phase results of the North American Olanzapine Trial. J Clin Psychopharmacol 21: 14-20.

Peters SR (2003a). The art of embedding tissue for frozen section. Part i: a system for face down cryoembedding of tissues using freezing temperature embedding wells. J Histotechnol 26: 11-19.

Peters SR (2003b). The art of embedding tissue for frozen section. Part ii: frozen block cryoembedding. J Histotechnol 26: 23-28.

Pierri JN, Chaudry AS, Woo TU, Lewis DA (1999). Alterations in chandelier neuron axon terminals in the prefrontal cortex of schizophrenic subjects. Am J Psychiatry 156: 1709-1719.

Rezaie P, Male D (1999). Colonisation of the developing human brain and spinal cord by microglia: a review. Microsc Res Technol 45: 359-382.

Scherle W (1970). A simple method for volumetry of organs in quantitative stereology. Mikroskopie 26: 57-60.

Selemon LD, Kleinman JE, Herman MM, Goldman-Rakic PS (2002). Smaller frontal gray matter volume in postmortem schizophrenic brains. Am J Psychiatry 159: 1983-1991.

Selemon LD, Lidow MS, Goldman-Rakic PS (1999). Increased volume and glial density in primate prefrontal cortex associated with chronic antipsychotic drug exposure. Biol Psychiatry 46: 161-172.

Selemon LD, Rajkowska G, Goldman-Rakic PS (1995). Abnormally high neuronal density in the schizophrenic cortex. A morphometric analysis of prefrontal area 9 and occipital area 17. Arch Gen Psychiatry 52: 805-818 (discussion 819-820).

Shenton ME, Dickey CC, Frumin M, McCarley RW (2001). A review of MRI findings in schizophrenia. Schizophr Res 49: 1-52.

Stark AK, Uylings HB, Sanz-Arigita E, Pakkenberg B (2004). Glial cell loss in the anterior cingulate cortex, a subregion of the prefrontal cortex, in subjects with schizophrenia. Am J Psychiatry 161: 882-888.

Sweet RA, Dorph-Petersen KA, Lewis DA (2005). Mapping auditory core, lateral belt, and parabelt cortices in the human superior temporal gyrus. J Comp Neurol 491: 270-289.

Szeszko PR, Goldberg E, Gunduz-Bruce H, Ashtari M, Robinson D, Malhotra AK et al (2003). Smaller anterior hippocampal formation volume in antipsychotic-naive patients with firstepisode schizophrenia. Am J Psychiatry 160: 2190-2197.

Thune JJ, Uylings HB, Pakkenberg B (2001). No deficit in total number of neurons in the prefrontal cortex in schizophrenics. J Psychiatr Res 35: 15-21.

Ukai W, Ozawa H, Tateno M, Hashimoto E, Saito T (2004). Neurotoxic potential of haloperidol in comparison with risperidone: implication of Akt-mediated signal changes by haloperidol. J Neural Transm 111: 667-681.

Wang C, McInnis J, Ross-Sanchez M, Shinnick-Gallagher P, Wiley JL, Johnson KM (2001). Long-term behavioral and neurodegenerative effects of perinatal phencyclidine administration: implications for schizophrenia. Neuroscience 107: 535-550.

Wei Z, Bai O, Richardson JS, Mousseau DD, Li XM (2003). Olanzapine protects PC12 cells from oxidative stress induced by hydrogen peroxide. J Neurosci Res 73: 364-368.

West MJ, Slomianka L, Gundersen HJ (1991). Unbiased stereological estimation of the total number of neurons in the subdivisions of the rat hippocampus using the optical fractionator. Anat Rec 231: 482-497.

Wood SJ, Velakoulis D, Smith DJ, Bond D, Stuart GW, McGorry PD et al (2001). A longitudinal study of hippocampal volume in first episode psychosis and chronic schizophrenia. Schizophr Res 52: $37-46$. 